\title{
Impacto de las condiciones de ingreso de pacientes a diálisis crónica sobre resultados clínicos y gasto sanitario durante el primer año de tratamiento
}

\author{
Santiago Torales ${ }^{1 *}$, Juan Altuna ${ }^{2}$ y Natalia Jorgënsen ${ }^{2}$ \\ ${ }^{1}$ Escuela Superior de Sanidad, Universidad Nacional del Litoral (ESS/UNL), Santa Fe; ${ }^{2}$ Instituto de Investigaciones Sanitarias de la Seguridad \\ Social (IISSS), Buenos Aires. Argentina
}

\begin{abstract}
Resumen
La enfermedad renal crónica presenta una prevalencia y morbimortalidad crecientes; en el primer año en diálisis ocurren la mayor cantidad de eventos y muertes, con costos incrementales. Objetivo: Establecer el efecto de las condiciones iniciales (clínicas y de cobertura) de los pacientes incidentes a diálisis en los eventos y sus costos durante el primer año de tratamiento. Métodos: Sobre una muestra retrospectiva (20,256 pacientes) en diálisis del sistema de salud argentino (2010-2015), seguida durante 1 año, se tomaron características epidemiológicas, clínicas y de cobertura, y se evaluó la morbimortalidad y costos asociados mediante análisis multivariables. Resultados: La mortalidad anual fue del $27 \%$ (66\% en el 1.er semestre). Comparado con el 2. ${ }^{\circ}$ semestre, hubo 5 veces más internaciones, duplicación de días/cama, el triple de consumo de prácticas, y costos 4 veces superiores, con diferencias entre financiadores y modelos de contratación. El gasto mensual/cápita fue hasta 45 veces superior en el 1 . $^{\text {er }}$ semestre: las variables asociadas fueron edad > 65 años, catéter transitorio al ingreso, albuminemia < $3 \mathrm{~g} / \mathrm{dl}$ y diagnóstico de diabetes, insuficiencia cardíaca y/o angor. Conclusiones: La población estudiada presentó una alta morbimortalidad, mayor consumo de servicios y costos en periodos cercanos al ingreso, asociados a variables clínicas y de cobertura conocidas, lo que evidencia una falta de seguimiento previo o selección inadecuada de pacientes.
\end{abstract}

Palabras clave: Fallo renal crónico. Diálisis renal. Factores epidemiológicos. Economía de la salud. Costos. Análisis de costo.

\section{Impact of initial conditions of chronic dialysis patients on clinical results and healthcare costs during the first year}

\section{Abstract}

Chronic kidney disease presents increasing prevalence, morbidity and mortality, with highest number of events, deaths and incremental costs during the first year in dialysis, Objective: To establish effect of initial conditions (clinical/coverage) of incident patients on dialysis for events and their costs in the first year of treatment. Methods: On a retrospective sample (20,256 patients) in dialysis from Argentinian health system (2010-2015) followed for a year, epidemiological, clinical and coverage characteristics were evaluated, assessing morbidity, mortality and associated costs through multivariate analysis.

\section{Correspondencia:}

*Santiago Torales

E-mail: santiago.torales@gmail.com Access bajo la licencia CC BY-NC-ND (http://creativecommons.org/licenses/by-nc-nd/4.0/)
Fecha de recepción: 04-02-2019

Fecha de aceptación: 08-07-2019

DOI: 10.24875/NEFRO.19000060
Disponible en internet: 09-08-2019

Nefro Latinoam. 2019;16:20-32 www.nefrologialatinoamericana.com 
Results: Annual mortality was $27 \%$ (66\% in $1^{\text {st }}$ semester). Compared to the $2^{\text {nd }}$ semester, there were 5 hospitalizations, double of days/bed, trice of practices, and costs 4 times higher, with differences between funders and hiring models. The monthly/ capita expenditure was up to 45 times higher in the $1^{\text {st }}$ semester: the associated variables were age $>65$ years, transient catheter on admission, albuminemia < $3 \mathrm{~g} / \mathrm{dl}$, and diagnosis of diabetes, heart failure and/or angina. Conclusions: This population showed high morbidity and mortality, higher consumption of services and costs in periods near to admission, associated with known clinical and coverage variables, evidencing a lack of prior follow-up or inadequate patient selection.

Key words: Chronic kidney failure. Kidney dialysis. Epidemiological factors. Health economics. Costs. Cost analysis.

\section{Introducción}

El ingreso del paciente con enfermedad renal crónica terminal (ERCT) a tratamiento con diálisis constituye una instancia crucial, donde usualmente se exponen las ineficiencias de los sistemas de salud en la detección temprana y el seguimiento previo ${ }^{1,2}$. Esto impide la preparación adecuada y de manera paradójica, los sistemas de atención despliegan allí enormes recursos y gastos destinados a garantizar la supervivencia del paciente y su adaptación a estos complejos tratamientos. Estudios observacionales muestran que en el año posterior al ingreso a diálisis se registran una gran cantidad de eventos clínicos, con particular preponderancia en el primer trimestre. A pesar de conocerse esta situación sanitaria, la evidencia internacional ${ }^{3,4}$ confirma tres realidades: que gran parte de los pacientes aún ingresan a diálisis sin seguimiento adecuado y articulado de los sistemas de atención clínica ${ }^{5}$; que esta falta de adecuación influye en los resultados de parámetros clínicos al ingreso; y que esta insuficiente preparación expone a los pacientes a mayor incidencia de eventos graves, principalmente cardiovasculares e infecciosos (fatales y no fatales), con un considerable impacto en la mortalidad, la pérdida de calidad de vida y el incremento de gastos de atención del sistema de salud ${ }^{6,7}$.

No hay en Latinoamérica suficientes publicaciones a gran escala de casos que demuestren esta relación entre el ingreso de los pacientes a diálisis y la cantidad de eventos, su gravedad y sus costos asociados. Muchas veces, esto obedece a diferentes modelos de financiamiento, con asimetría en la provisión y compra de servicios de salud, fragmentación de la atención y la escasa disponibilidad de la información para relacionar estas instancias: un caso paradigmático podría ser el de Argentina, con un sistema donde participan más de 350 entes de financiamiento de prácticas sanitarias, con dispar generación de información clínica y administrativa.

Aun existiendo una elevada prevalencia de la diabetes y la hipertensión arterial como causas de la ERCT, muchos de estos pacientes (hasta el $50 \%$ en algunas series) continúan llegando a diálisis sin recibir una consulta 0 valoración nefrológica previa ${ }^{8}$. Las consecuencias de las referencias tardías influyen en el aumento de la morbilidad, la mortalidad, la calidad de vida y la mayor utilización de los recursos. Una revisión del estudio DOPPS encontró que el riesgo de muerte fue elevado durante los primeros 120 días en comparación al periodo de 121 a 365 días (27.5 vs. 21.9 muertes por cada 100 años/paciente; $p=0.002$ ). Las tasas de mortalidad por causa específica fueron más altas en los primeros 120 días que en los siguientes 121 a 365 días para casi todas las causas (la principal fue la muerte de causa cardiovascular) ${ }^{9}$. Los pacientes con atención de diálisis crónica incurren en importantes recursos sanitarios debido a los costos de la diálisis y las complicaciones. Los reportes sobre el impacto económico y la inequidad distributiva de la ERC avanzada (estadíos 3 a 5) son evidentes y generan alarma en los sistemas de financiamiento ${ }^{10}$.

La prevalencia en la Argentina de ERCT con tratamiento dialítico es de 664 pacientes por millón de habitantes, con una incidencia anual de 163 pacientes por millón de habitantes y se evidencian diferencias entre provincias y regiones sanitarias, principalmente a través de hemodiálisis (93.8\%), de acuerdo a los reportes del Registro SINTRA.SAN/INCUCAI ${ }^{11}$. Tanto la prevalencia como la incidencia de ERCT continúan incrementándose en el país $(2.1 \%$ y $1.5 \%$ anual, respectivamente), pero los resultados clínicos de los pacientes al ingreso son malos: 2/3 presentan catéteres transitorios y la mitad se encuentra con malnutrición o síndrome anémico. Un factor potencial implicado en estos malos resultados es la fragmentación de los sistemas de atención, gestión clínica y financiamiento de prestaciones, así como las reconocidas asimetrías de compra de servicios y oferta sanitaria existentes entre distintas coberturas y jurisdicciones ${ }^{12}$. Esta combinación de situaciones puede afectar tanto los resultados de los pacientes (por diferente accesibilidad a los sistemas de atención) como al gasto acumulativo, 
lo que genera un área de estudio sobre la eficiencia y la equidad en el acceso a cuidados de calidad sanitaria.

Considerando este contexto, el objetivo del presente trabajo es determinar la relación entre la situación de las variables (clínicas y de cobertura) al ingreso a diálisis de una población representativa de pacientes renales crónicos terminales incidentes en Argentina, con la aparición de eventos durante el primer año en tratamiento, su gravedad y gastos sanitarios acumulativos, más un impacto diferencial según la modalidad de contratación.

\section{Materiales y métodos}

Se planteó un estudio descriptivo y cuantitativo, con base en datos epidemiológicos y de consumos prestacionales retrospectivos provenientes de financiadores relevantes de la seguridad social en Argentina, para intentar establecer la existencia de correlaciones y su magnitud entre la característica epidemiológicas, la situación clínica y la cobertura de ingreso (como variables independientes) de los pacientes con ERCT a tratamiento dialítico, y la aparición de eventos, su consumo de servicios y el gasto acumulativo (como variables dependientes) de los mismos en el periodo del año posterior a la fecha de ingreso. Se efectuaron análisis univariados buscando tendencias de distribución y multivariables para evaluar magnitud diferencial de efecto entre ellas.

\section{Variables}

\section{EPIDEMIOLÓGICAS}

- Edad en cuatro distribuciones (18-44 años, 45-64 años, 65-74 años y > 74 años).

- Género (masculino/femenino).

- Enfermedad de base (diabetes/no diabetes).

- Comorbilidades cardiovasculares (angina persistente y/o insuficiencia cardíaca al ingreso).

\section{Clínicas}

- Acceso vascular (fístula nativa/prótesis/catéter HD transitorio + permanente).

- Hematocrito (menos de 22\%, 22 a 26\%, 26 a $30 \%$ y más de $30 \%$ ).

- Albuminemia (menos de $3 \mathrm{~g} / \mathrm{dl}$, 3 a $4 \mathrm{~g} / \mathrm{dl}$ y más de $4 \mathrm{~g} / \mathrm{dl})$.

\section{Cobertura}

- Seguro (Obra Social Provincial [OSP]/Obra Social Nacional [OSN]/Programa Asistencial Médico Integral [PAMI).

- Esquema de contratación (prestación/cápita).

\section{Consumos PRESTACIONALES}

- Ambulatorios (consultas, bioquímica, prácticas diagnósticas y terapéuticas).

- Internaciones (número, días cama, clínica/quirúrgica).

- Sesiones de diálisis (número total, tiempo en tratamiento).

\section{GASTO PRESTACIONAL}

Se tomaron los valores unitarios de cada cobertura y prestación seleccionada vigentes a noviembre de 2017, combinándose con los consumos prestacionales para obtener registros del costo acumulado a través de la siguiente formulación:

suma de tasas de utilización de prácticas $\times$ valor unitario de prácticas = gasto acumulado por paciente (en $A R \$$ ).

Para su análisis más ajustado, estos consumos globales y gastos acumulados se discriminaron en dos momentos definidos, tanto en forma global como entre coberturas: $1 .^{\text {er }}$ trimestre vs. $4 .^{\circ}$ trimestre y $1 .^{\text {er }}$ semestre vs. $2 .^{\circ}$ semestre.

\section{Población y muestra}

Sobre un total de 27,966 pacientes en diálisis en Argentina (Registro de Diálisis Crónica 2013, SAN-INCUCAI), el $80.2 \%$ de ellos pertenecen a la Seguridad Social, donde encontramos al PAMI, las OSN y las OSP. Se consideraron pacientes con ERCT incidentes durante el periodo de enero de 2010 a diciembre de 2015 bajo estas coberturas, con seguimiento de los eventos y registro de consumos de prestaciones durante el año calendario siguiente a la fecha de ingreso a diálisis. El tamaño muestral estimado fue de 3,387 pacientes (nivel de confianza $95 \%$, nivel de error $1 \%$ y pérdida estimada $50 \%$ ). La información del listado obtenido fue anonimizado siguiendo la identificación por número de DNI y con enmascaramiento del tipo de cobertura; luego, se realizó el apareamiento del listado para los consumos de historias prestacionales retrospectivas en las bases transaccionales de los financiadores. La información se consolidó al final del proceso, con intervención de los coordinadores de la investigación. 
Tabla 1. Variables descriptivas de la muestra total

\begin{tabular}{|c|c|c|c|c|}
\hline Variables comparativas & Total & PAMI & OSN & OSP \\
\hline $\begin{array}{l}\text { Generales } \\
\text { Hombre }(\%) \\
\text { Edad promedio } \\
\text { Diabéticos }(\%)\end{array}$ & $\begin{array}{c}58.50 \% \\
66.24( \pm 5.2) \\
41.92 \%\end{array}$ & $\begin{array}{c}58.27 \% \\
68.42( \pm 4.1) \\
42.71 \%\end{array}$ & $\begin{array}{c}58.29 \% \\
50.53( \pm 8.2) \\
26.20 \%\end{array}$ & $\begin{array}{c}59.94 \% \\
58.27( \pm 6.3) \\
41.77 \%\end{array}$ \\
\hline $\begin{array}{l}\text { Acceso vascular } \\
\text { Catéter transitorio (\%) } \\
\text { Fístula nativa (\%) } \\
\text { Fístula protésica (\%) } \\
\text { Catéter semipermanente (\%) }\end{array}$ & $\begin{array}{l}70.54 \% \\
24.87 \% \\
3.04 \% \\
1.55 \%\end{array}$ & $\begin{array}{l}70.31 \% \\
25.10 \% \\
3.10 \% \\
1.49 \%\end{array}$ & $\begin{array}{l}79.01 \% \\
17.11 \% \\
1.74 \% \\
2.14 \%\end{array}$ & $\begin{array}{l}69.28 \% \\
25.92 \% \\
3.02 \% \\
1.78 \%\end{array}$ \\
\hline $\begin{array}{l}\text { Albuminemia } \\
\text { Menor a } 3 \mathrm{~g} / \mathrm{l} \\
\text { Entre } 3 \text { y } 4 \mathrm{~g} / \mathrm{l} \\
\text { Mayor a } 4 \mathrm{~g} / \mathrm{l}\end{array}$ & $\begin{array}{l}21.95 \% \\
45.58 \% \\
32.48 \%\end{array}$ & $\begin{array}{l}22.62 \% \\
46.34 \% \\
31.05 \%\end{array}$ & $\begin{array}{l}19.79 \% \\
46.26 \% \\
33.96 \%\end{array}$ & $\begin{array}{l}18.76 \% \\
41.04 \% \\
40.20 \%\end{array}$ \\
\hline $\begin{array}{l}\text { Hematoc rito } \\
\text { Inferior a } 22 \% \\
\text { Entre } 22 \text { y } 25 \% \\
\text { Entre } 25 \text { y } 30 \% \\
\text { Mayor a } 30 \%\end{array}$ & $\begin{array}{c}9.93 \% \\
21.37 \% \\
35.25 \% \\
33.44 \%\end{array}$ & $\begin{array}{l}9.96 \% \\
21.36 \% \\
35.06 \% \\
33.62 \%\end{array}$ & $\begin{array}{l}11.63 \% \\
20.59 \% \\
38.64 \% \\
29.14 \%\end{array}$ & $\begin{array}{l}9.23 \% \\
21.56 \% \\
35.55 \% \\
33.66 \%\end{array}$ \\
\hline $\begin{array}{l}\text { Comorbilidad cardiovascular } \\
\text { Angina persistente } \\
\text { Insuficiencia cardíaca }\end{array}$ & $\begin{array}{l}11.22 \% \\
22.87 \%\end{array}$ & $\begin{array}{l}11.05 \% \\
23.35 \%\end{array}$ & $\begin{array}{l}10.03 \% \\
17.91 \%\end{array}$ & $\begin{array}{l}12.54 \% \\
21.66 \%\end{array}$ \\
\hline Suma de pacientes & 20,256 & 16,538 & 823 & 2,895 \\
\hline Fallecidos en el primer año (\%) & $26.04 \%$ & $27.25 \%$ & $17.38 \%$ & $21.55 \%$ \\
\hline
\end{tabular}

OSN: Obra Social Nacional; OSP: Obra Social Provincial; PAMI: Programa Asistencial Médico Integral.

\section{Análisis estadístico}

Las variables identificadas se introdujeron secuencialmente en análisis univariante y luego multivariante, para identificar el posible efecto parcial sobre las probabilidades proporcionales de Cox (nivel de significación estadística/riesgo alfa $=5 \%$ ). Los resultados como tiempo al evento «mortalidad» se expresaron como hazard ratios. Finalmente, para evaluar las variables condicionantes del evento «internación", se utilizó un modelo de regresión logístico (presencia $=10$ ausencia $=0$ del evento). Las covariables fueron las mismas del modelo de sobrevida global. Los resultados de ocurrencia del evento se expresaron como odds ratios. Se utilizaron los programas Microsoft Exce ${ }^{\circledR}$ y Stata ${ }^{\circledR} 14$.

\section{Resultados}

\section{Análisis descriptivo de la información}

Se tomó la información de 8 instituciones (1 PAMI, 5 OSP y 3 OSN), y se superaron los tamaños muestrales planificados: 20,256 observaciones para las estimaciones de morbimortalidad (PAMI: 16,538 pacientes
$81.6 \%$ ], OSN: $823{ }^{4} .1 \%$ ], y OSP: $2,895^{14} .3 \%$ ]) y 3,711 observaciones para las estimaciones de tasas de utilización y costos (PAMI: 2,631 pacientes 70.9\%] y el resto de las obras sociales: 1,080).

Teniendo en cuenta que la población del PAMI abarca principalmente a la población jubilada y que la muestra presenta marcadas diferencias en la cantidad de pacientes entre coberturas, se realizó un análisis distinguiendo por tipo de financiador. En la tabla 1 se presenta una descripción de las características de las tres poblaciones.

Como puntos relevantes, casi un $60 \%$ de la población es de sexo masculino. La edad promedio en el PAMl fue de 68 años (significativamente más elevada) vs. 50 años en las OSN y 58 años en las OSP $(p<0.005)$. La prevalencia de diabetes fue similar entre el PAMI y las OSP, alrededor de un $42 \%$, mientras que para las OSN fue del $26 \%(p<0.005)$. El acceso vascular más frecuente fue el catéter (transitorio + semipermanente), con valores similares entre el PAMl y las OSP $(70 \%)$, y más elevada en el caso de las OSN (79\%): el siguiente acceso vascular más frecuente fue la fístula arteriovenosa nativa (PAMl y OSP con un 25\% 


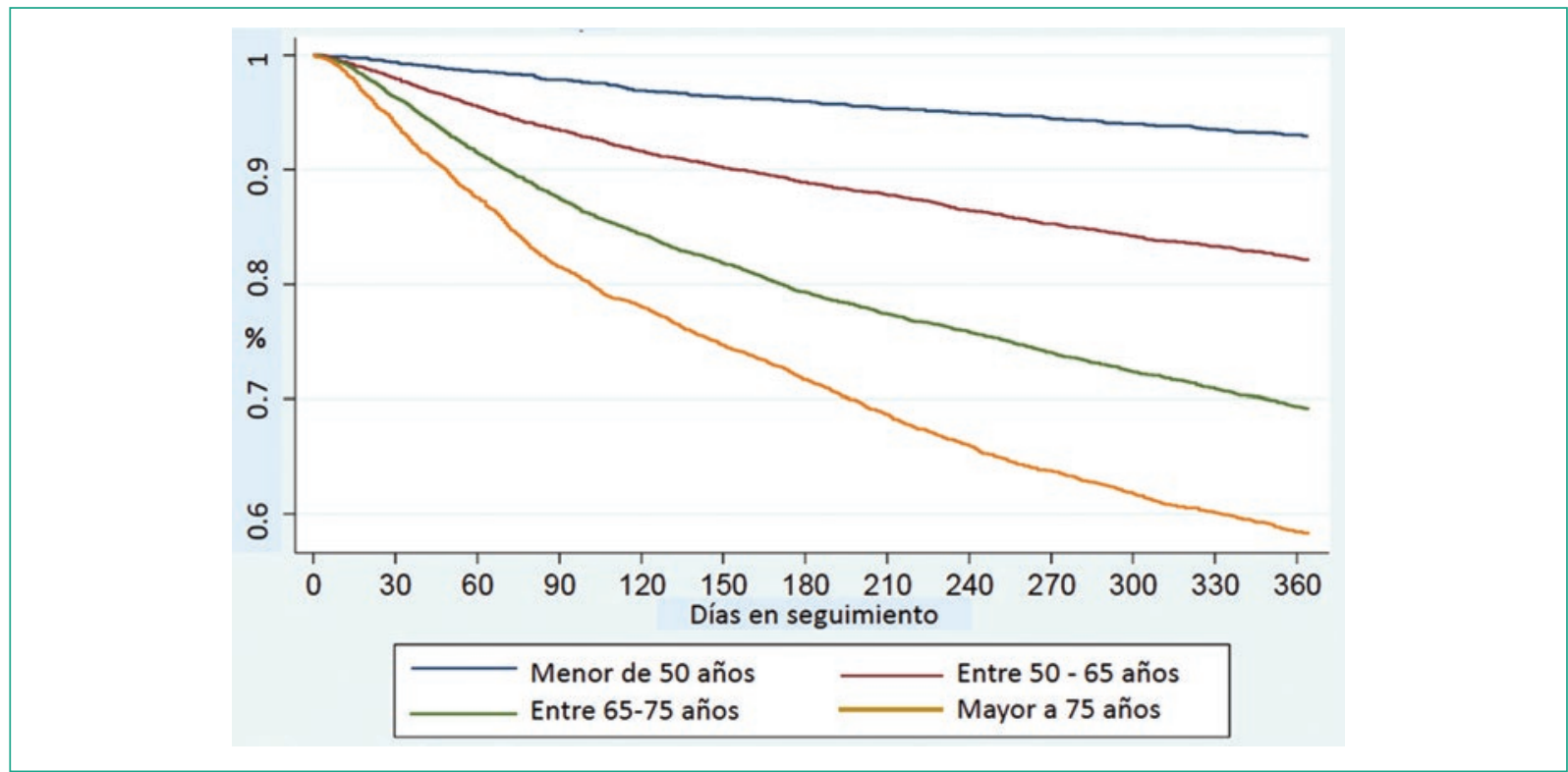

Figura 1. Curvas de sobrevida (Kaplan Meier) por edad (<50; 50-65; 65-75, y > 75 años).

Tabla 2. Mortalidad total en el primer año (por trimestres y por tipo de financiador)

\begin{tabular}{|c|c|c|c|c|}
\hline $\begin{array}{l}\text { Mortalidad por } \\
\text { trimestre }\end{array}$ & PAMI & OSP & OSN & Total \\
\hline \multirow[t]{2}{*}{ 1. er trimestre } & 1,906 & 243 & 55 & 2,204 \\
\hline & $42.29 \%$ & $38.94 \%$ & $38.46 \%$ & $41.79 \%$ \\
\hline \multirow[t]{2}{*}{$2 .^{\circ}$ trimestre } & 1,148 & 148 & 38 & 1,334 \\
\hline & $25.47 \%$ & $23.72 \%$ & $26.57 \%$ & $25.29 \%$ \\
\hline \multirow[t]{2}{*}{ 3. er trimestre $^{2}$} & 793 & 125 & 32 & 950 \\
\hline & $17.59 \%$ & $20.03 \%$ & $22.38 \%$ & $18.01 \%$ \\
\hline \multirow[t]{2}{*}{ 4. ${ }^{\circ}$ trimestre } & 660 & 108 & 18 & 786 \\
\hline & $14.64 \%$ & $17.31 \%$ & $12.59 \%$ & $14.90 \%$ \\
\hline \multirow[t]{2}{*}{ Total } & 4,507 & 624 & 143 & 5,274 \\
\hline & $27.25 \%$ & $17.38 \%$ & $21.55 \%$ & $26.04 \%$ \\
\hline
\end{tabular}

OSN: Obra Social Nacional; OSP: Obra Social Provincial; PAMI: Programa Asistencial Médico Integral.

de casos y OSN con un 17\%). El nivel de albúmina fue similar en los tres financiadores, con un porcentaje de desnutrición (valor menor a $3 \mathrm{~g} / \mathrm{dl}$ ) cercano al $20 \%$. De acuerdo al valor de hematocrito, en cuatro rangos de agrupación, se observó una distribución homogénea entre financiadores: en los tres casos, un $10 \%$ de los pacientes se encontraba por debajo del $22 \%$.

\section{Análisis de mortalidad}

\section{ANÁLISIS UNIVARIANTE}

En el primer año de tratamiento se registraron 5,274 muertes en los 20,256 pacientes evaluados (26.04\%). Analizando por periodos trimestrales, la frecuencia de muertes se muestra muy elevada en los primeros 90 días, y disminuye trimestre a trimestre (Tabla 2). El comportamiento de cada variable se observa en los gráficos:

- Edad: Los pacientes entre 50 y 65 años presentaron una probabilidad de sobrevida al año $>80 \%$; a partir de ese límite, las probabilidades caen de forma pronunciada, especialmente en los primeros 90 días, y luego caen de forma menos brusca, con una probabilidad de sobrevida menor al $70 \%$ para pacientes entre 65 y 75 años y menor al $60 \%$ para pacientes mayores de 75 años: al año de tratamiento, 4 de cada 10 pacientes $>75$ años han fallecido (Fig. 1).

- Diabetes: La sobrevida es similar a lo largo de los primeros trimestres, se establecen las primeras diferencias significativas después del $9 .^{\circ}$ mes de tratamiento (Fig. 2).

- Comorbilidad cardiovascular: La presencia de angina persistente muestra una influencia marcada en la curva de sobrevida desde el primer trimestre de tratamiento (aun con un número bajo de pacientes). La insuficiencia cardíaca fue más frecuente, y evidenció 


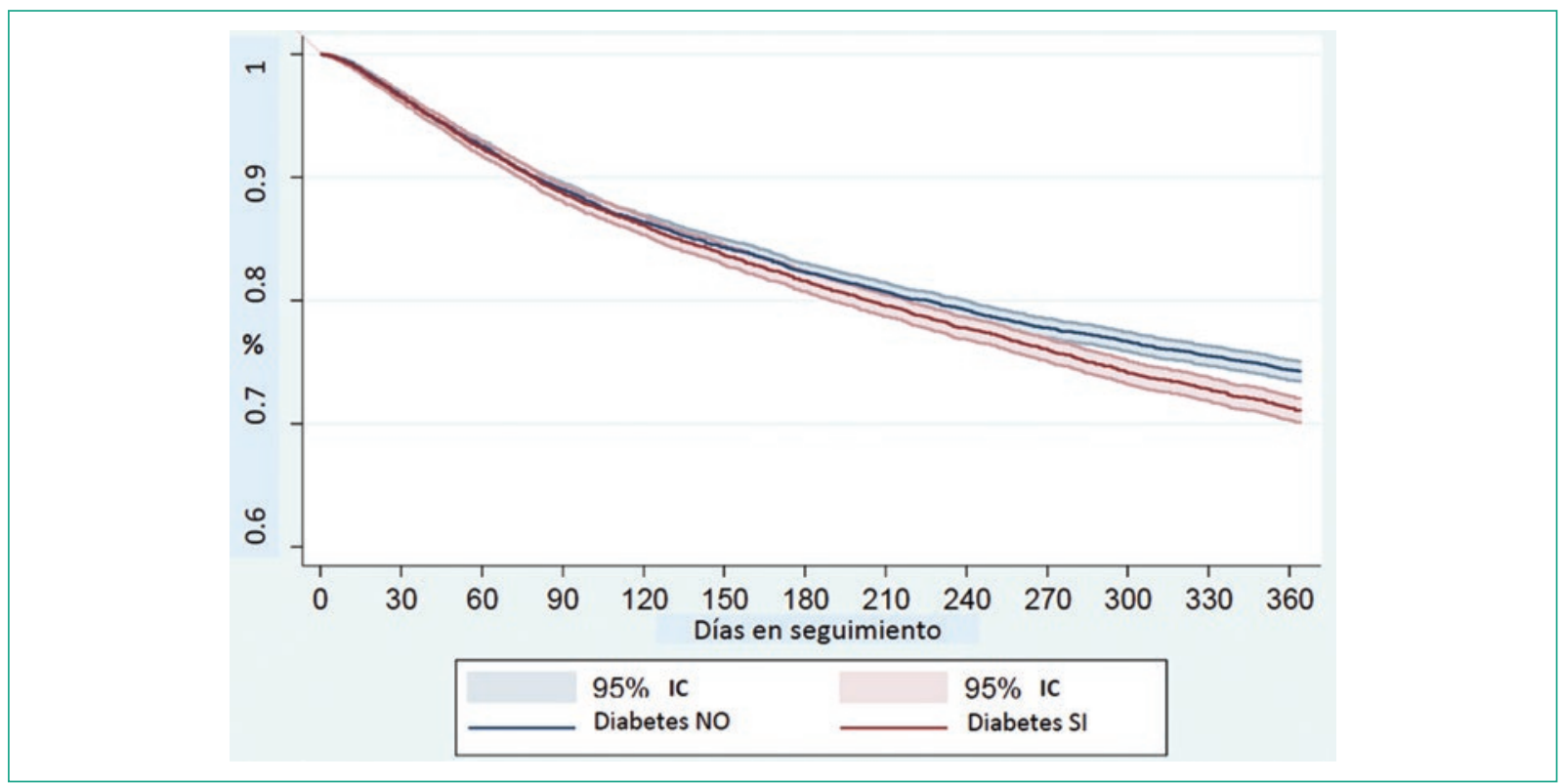

Figura 2. Curvas de sobrevida (Kaplan Meier) por diagnóstico de diabetes.

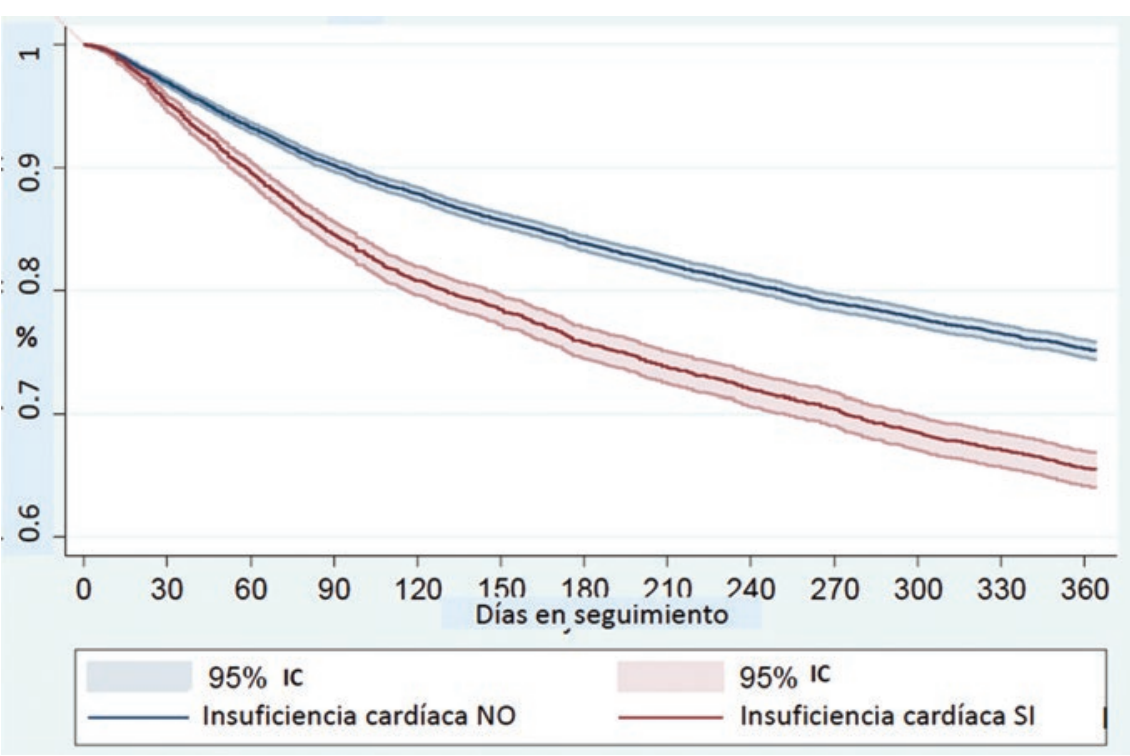

Figura 3. Curvas de sobrevida (Kaplan Meier) por insuficiencia cardíaca.

también un impacto temprano y sostenido en la mortalidad en la población observada (Fig. 3).

- Acceso vascular: Los pacientes con fístula nativa o protésica al ingreso a diálisis presentan mayor sobrevida (entre el 80 y el $85 \%$ ) con respecto a aquellos con catéter transitorio y semipermanente, $<70 \%$ en ambos casos (sobre 10 pacientes ingresados con catéter, $>3$ fallecen antes del año) (Fig. 4).
- Albuminemia: Se observó un impacto marcado con niveles $<3 \mathrm{~g} / \mathrm{dl}$. Casi 4 sobre 10 pacientes que ingresan con estos cuadros de desnutrición grave mueren antes del año (Fig. 5).

- Tipo de cobertura: La población en diálisis de las OSN presenta una probabilidad de sobrevida del $82 \%$ frente a las OSP (78\%) y el PAMI (72\%). A los 90 días, las probabilidades de sobrevida decrecen 


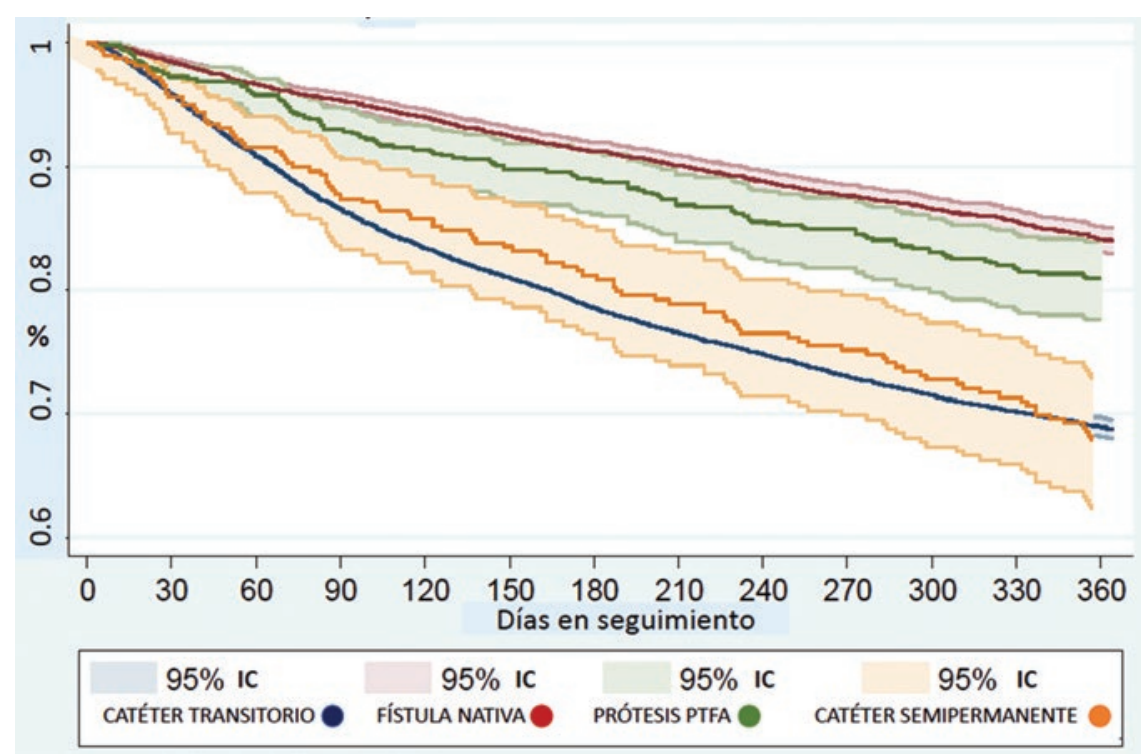

Figura 4. Curvas de sobrevida (Kaplan Meier) por tipo de acceso vascular.

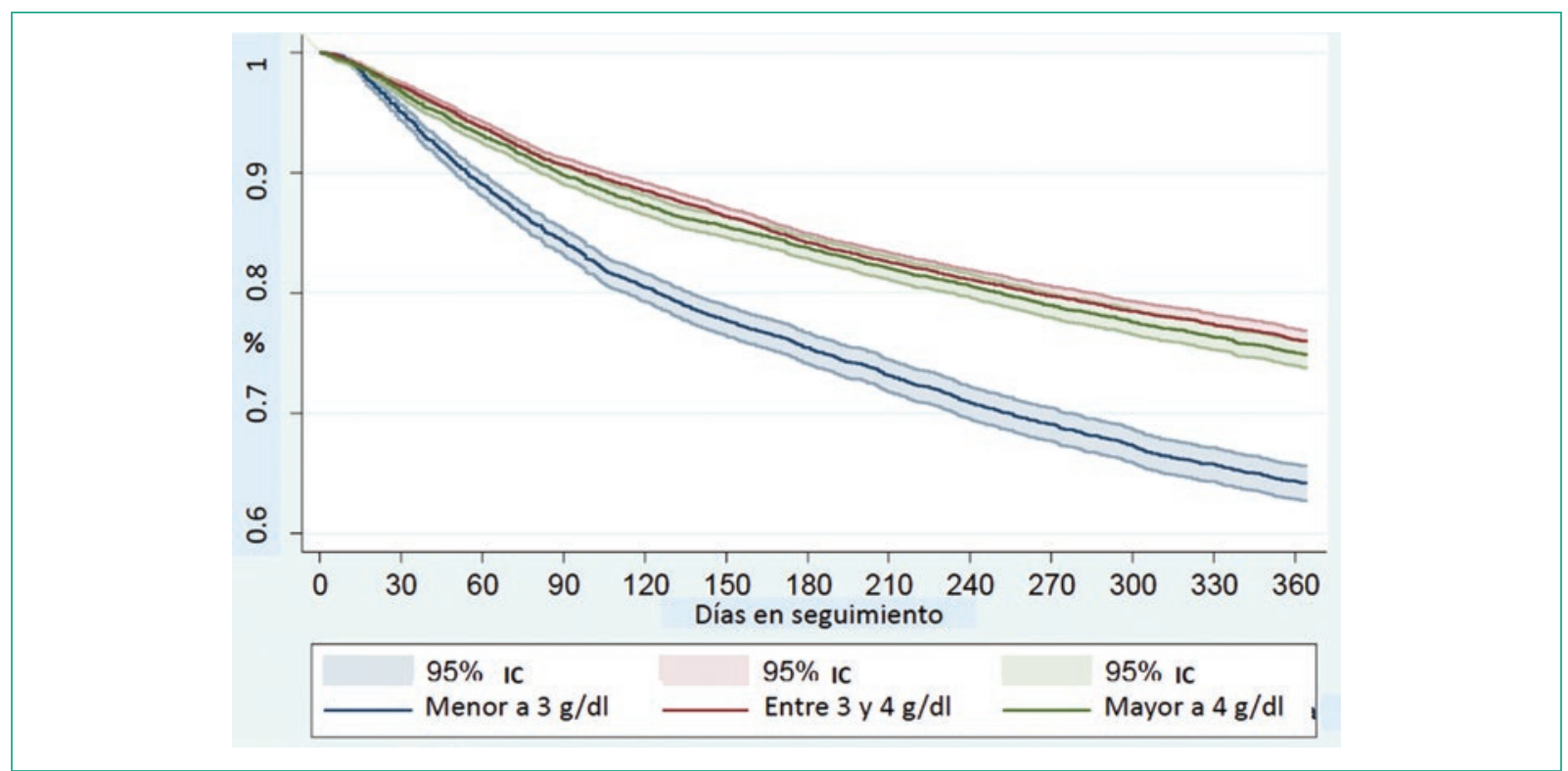

Figura 5. Curvas de sobrevida (Kaplan Meier) por niveles de albuminemia (g/dl).

levemente, pero la brecha entre el PAMI y las OS comienza a aumentar, en el primer año de tratamiento fallecen 3 de cada 10 pacientes PAMI ingresados, y 2 de ellos en los primeros 180 días (Fig. 6).

\section{ANÁLISIS MULTIVARIANTE}

Se generó un caso base a partir de datos seleccionados: paciente mujer, menor de 50 años, no diabética, con catéter transitorio, hematocrito $<22 \%$, albuminemia $<4 \mathrm{~g} / \mathrm{dl}$, sin angina, sin insuficiencia cardíaca, en hemodiálisis, con Obra Social Provincial y residente en la región Centro. Los resultados globales se muestran en la tabla 3.

Sin diferencias en la distribución por sexo, se observa el marcado efecto en las cuatro franjas etarias definidas, siendo muy superior la prevalencia de mortalidad en los > 75 años (riesgo de mortalidad 5 veces 


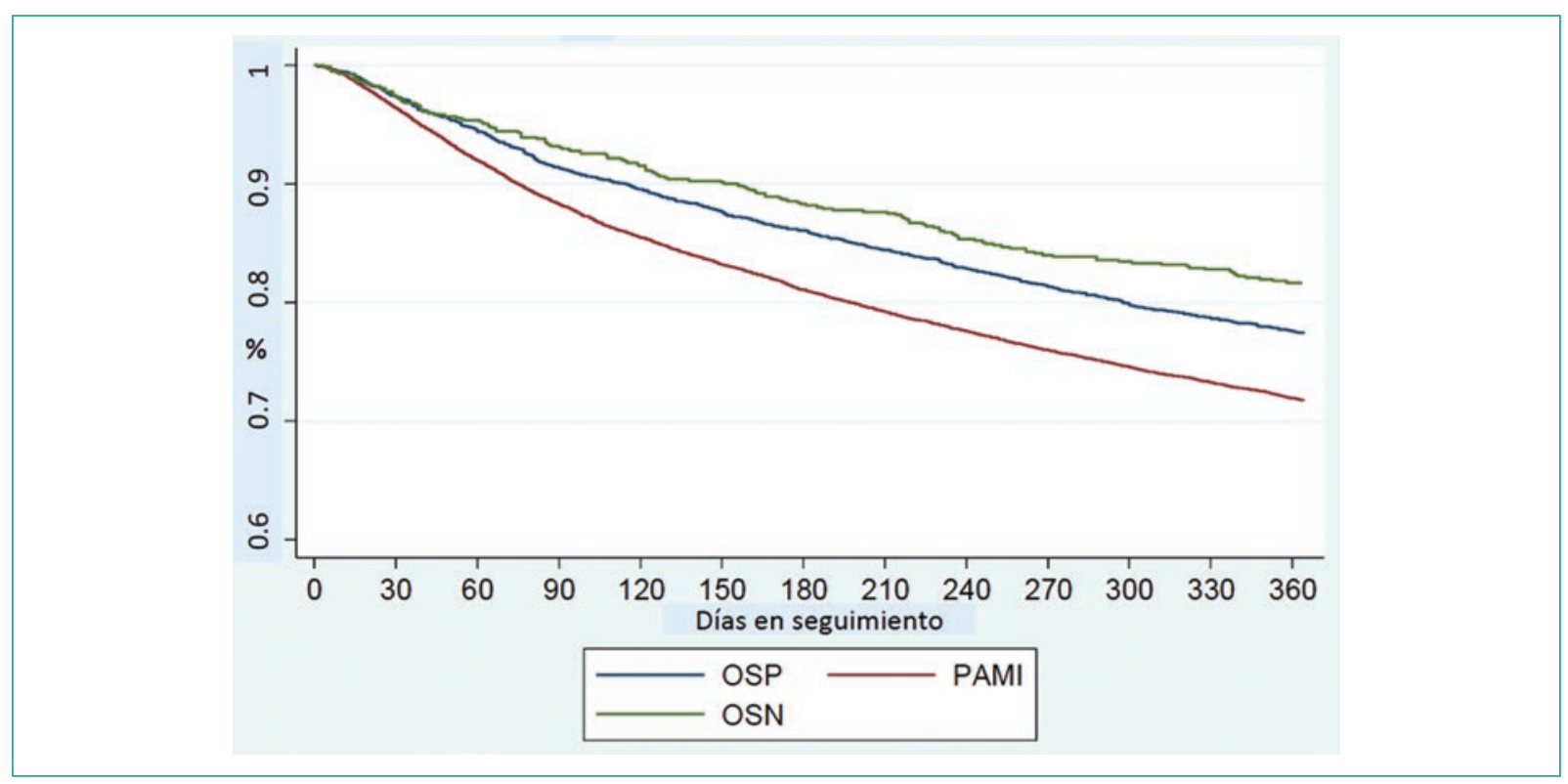

Figura 6. Curvas de sobrevida (Kaplan Meier) por tipo de cobertura.

OSN: Obra Social Nacional; OSP: Obra Social Provincial; PAMI: Programa Asistencial Médico Integral.

superior). La diabetes representa un $25 \%$ más de probabilidad significativa de muerte. En cuanto al tipo de acceso vascular, los pacientes con catéter (transitorio + semipermanente) tienen un $33 \%$ más de probabilidad de muerte que aquellos con fístula nativa y un $24 \%$ más de probabilidad de fallecer en el primer año que un paciente con fístula protésica (ambos valores significativos). Se encontró una clara asociación entre la mortalidad y la albuminemia $<3 \mathrm{~g} / \mathrm{dl}$ : ingresar a diálisis en esta condición presenta un significativo riesgo de mortalidad (22\% superior). A diferencia de otras series poblacionales, no hubo influencia observable del hematocrito al ingreso con la mortalidad anual entre las cuatro bandas o rangos de valores definidos. En cuanto a la influencia de las comorbilidades cardiovasculares, el diagnóstico de angina persistente genera un 9\% mayor de riesgo y la insuficiencia cardíaca un 17\% más de mortalidad, siendo estos dos valores significativos. Ajustando por todas las variables, no hubo diferencias significativas en mortalidad entre las coberturas analizadas.

\section{Análisis de consumos y gastos prestacionales}

Sobre una base de 3,345 registros, se analizaron comparativamente los resultados de consumo promedio por paciente en el PAMI (2,641 pacientes, sistema per cápita), las OSN y las OSP sumadas (1,080 pacientes, sistema por prestaciones), y se discriminó en los periodos: $1 .^{\text {er }}$ trimestre vs. $4 .^{\circ}$ trimestre y $1 .^{\text {er }}$ semestre vs. $2 .^{\circ}$ semestre.

\section{ANÁLISIS UNIVARIANTE}

Prácticas ambulatorias: Se consideraron las consultas clínicas, las prácticas bioquímicas, las prácticas ambulatorias diagnósticas y terapéuticas. Se encontraron claras diferencias, con mayor concentración y frecuencia del número de prestaciones en los primeros meses de tratamiento, tanto para las consultas $\left(+156 \%\right.$ en el $1 .^{\text {er }}$ vs. el $2 .^{\circ}$ semestre) como bioquímica $\left(+160 \%\right.$ en el $1 .^{\text {er }}$ vs. el $4 .^{\circ}$ trimestre, $p<0.005$ ). Las diferencias más marcadas (todas en rango significativo) se observaron en las prácticas diagnósticas (+323\% en el $1{ }^{\text {er }}$ vs. el $4 .{ }^{\circ}$ trimestre) y las prácticas terapéuticas nomencladas (2.11 prácti-

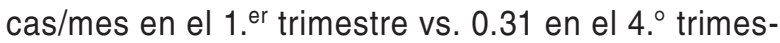
tre,$+713 \%)$.

Internaciones: Los eventos de internación fueron considerados como la mejor muestra disponible de impacto en la morbilidad de las variables al ingreso en tratamiento. Los valores fueron expresados como la suma de eventos totales en cada subgrupo de cobertura y como la aparición del evento en relación a la cantidad de días o meses (Tabla 4). 
Nefro Latinoam. 2019;16

Tabla 3. Regresión de riesgos proporcionales de Cox en hazard ratios para mortalidad

\begin{tabular}{|c|c|c|c|c|c|c|}
\hline CASO BASE (en negrita) & HR & SE & $z$ & $p>z$ & \multicolumn{2}{|c|}{ IC $95 \%$} \\
\hline $\begin{array}{l}\text { Mujer } \\
\text { Hombre }\end{array}$ & 1.03 & 0.02 & 1.33 & 0.18 & 0.99 & 1.06 \\
\hline $\begin{array}{l}\text { Edad (menor } 50 \text { años) } \\
\text { Entre } 50 \text { y } 65 \text { años } \\
\text { Entre } 65 \text { y } 75 \text { años } \\
\text { Mayor de } 75 \text { años }\end{array}$ & $\begin{array}{l}2.38 \\
3.78 \\
5.43\end{array}$ & $\begin{array}{l}0.13 \\
0.20 \\
0.29\end{array}$ & $\begin{array}{l}15.91 \\
24.78 \\
31.36\end{array}$ & $\begin{array}{l}0.00 \\
0.00 \\
0.00\end{array}$ & $\begin{array}{l}2.14 \\
3.40 \\
4.89\end{array}$ & $\begin{array}{l}2.65 \\
4.20 \\
6.04\end{array}$ \\
\hline $\begin{array}{c}\text { No diabético } \\
\text { Diabético }\end{array}$ & 1.25 & 0.02 & 11.31 & 0.00 & 1.20 & 1.29 \\
\hline $\begin{array}{l}\text { Catéter transitorio } \\
\text { Fístula nativa } \\
\text { Fístula protésica } \\
\text { Catéter semipermanente }\end{array}$ & $\begin{array}{l}0.67 \\
0.76 \\
0.94\end{array}$ & $\begin{array}{l}0.01 \\
0.04 \\
0.07\end{array}$ & $\begin{array}{l}-17.88 \\
-5.33 \\
-0.90\end{array}$ & $\begin{array}{l}0.00 \\
0.00 \\
0.37\end{array}$ & $\begin{array}{l}0.64 \\
0.69 \\
0.81\end{array}$ & $\begin{array}{l}0.70 \\
0.84 \\
1.08\end{array}$ \\
\hline $\begin{array}{l}\text { Albuminemia menor de } 3 \mathrm{~g} / \mathrm{l} \\
\text { Entre } 3 \text { y } 4 \mathrm{~g} / \mathrm{l} \\
\text { Mayor a } 4 \mathrm{~g} / \mathrm{l}\end{array}$ & $\begin{array}{l}0.78 \\
0.79\end{array}$ & $\begin{array}{l}0.02 \\
0.02\end{array}$ & $\begin{array}{l}-10.30 \\
-8.88\end{array}$ & $\begin{array}{l}0.00 \\
0.00\end{array}$ & $\begin{array}{l}0.75 \\
0.76\end{array}$ & $\begin{array}{l}0.82 \\
0.84\end{array}$ \\
\hline $\begin{array}{l}\text { Hematocrito menor del } 22 \% \\
\text { Entre } 22 \text { y } 26 \% \\
\text { Entre } 26 \text { y } 30 \% \\
\text { Mayor a } 30 \%\end{array}$ & $\begin{array}{l}0.96 \\
0.96 \\
0.96\end{array}$ & $\begin{array}{l}0.03 \\
0.03 \\
0.03\end{array}$ & $\begin{array}{l}-1.02 \\
-1.31 \\
-1.15\end{array}$ & $\begin{array}{l}0.31 \\
0.19 \\
0.25\end{array}$ & $\begin{array}{l}0.90 \\
0.90 \\
0.90\end{array}$ & $\begin{array}{l}1.03 \\
1.02 \\
1.03\end{array}$ \\
\hline $\begin{array}{l}\text { Sin angor ni ICC } \\
\text { Angina persistente } \\
\text { Insuficiencia cardíaca }\end{array}$ & $\begin{array}{l}1.09 \\
1.17\end{array}$ & $\begin{array}{l}0.03 \\
0.03\end{array}$ & $\begin{array}{l}3.02 \\
7.26\end{array}$ & $\begin{array}{l}0.00 \\
0.00\end{array}$ & $\begin{array}{l}1.03 \\
1.12\end{array}$ & $\begin{array}{l}1.15 \\
1.22\end{array}$ \\
\hline
\end{tabular}

HR: hazard ratio; ICC: insuficiencia cardíaca congestiva; SE: error estándar.

Tabla 4. Suma de eventos de internación y frecuencia, comparaciones entre coberturas

\begin{tabular}{|c|c|c|c|c|c|c|}
\hline Periodo & Cobertura & Pacientes & $\begin{array}{l}\text { Suma días en } \\
\text { diálisis }\end{array}$ & $\begin{array}{l}\text { Suma eventos } \\
\text { internación }\end{array}$ & $\begin{array}{c}\text { Evento internación } \\
c / X \text { días }\end{array}$ & $\begin{array}{l}\text { Evento internación } \\
\text { c/X meses }\end{array}$ \\
\hline \multirow[t]{2}{*}{ GENERAL } & PAMI & 2,641 & 846,168 & 3653 & 231.6 & 7.7 \\
\hline & Otras OS & 1,080 & 338,602 & 2177 & 155.5 & 5.2 \\
\hline \multirow[t]{2}{*}{ 1. ${ }^{\text {er }}$ trimestre } & PAMI & 212 & 13,056 & 297 & 44.0 & 1.5 \\
\hline & Otras OS & 102 & 6,756 & 209 & 32.3 & 1.1 \\
\hline \multirow[t]{2}{*}{$4 .^{\circ}$ trimestre } & PAMI & 2,152 & 781,935 & 2680 & 291.8 & 9.7 \\
\hline & Otras OS & 860 & 310,022 & 1687 & 183.8 & 6.1 \\
\hline \multirow[t]{2}{*}{$1{ }^{\text {er }}$ semestre } & PAMI & 358 & 34,377 & 626 & 54.9 & 1.8 \\
\hline & Otras OS & 168 & 17,561 & 376 & 46.7 & 1.6 \\
\hline \multirow[t]{2}{*}{$2 .^{\circ}$ semestre } & PAMI & 2,278 & 811,790 & 3027 & 268.2 & 8.9 \\
\hline & Otras OS & 909 & 321,138 & 1806 & 177.8 & 5.9 \\
\hline
\end{tabular}

OS: obras sociales; PAMI: Programa Asistencial Médico Integral.

En términos generales, los eventos de internación fueron significativamente más frecuentes en otras OS (un evento cada 5.2 meses) vs. el PAMl (1 evento cada 7.7 meses) y esta tendencia se repitió significativamente en los periodos más precoces en ambas coberturas: 1 evento cada 1.8 meses en el $1 .^{\text {er }}$ semestre vs. 8.9 meses en el $2 .^{\circ}$ trimestre para el PAMI, y 1 evento cada 1.6 meses en el $1 .^{\text {er }}$ semestre 
Tabla 5. Promedio de gasto acumulado total y mensual + incremento del gasto extradiálisis por paciente, comparación entre periodos

\begin{tabular}{|c|c|c|c|c|c|c|c|}
\hline Cobertura & Periodo & $\begin{array}{l}\text { Número de } \\
\text { pacientes }\end{array}$ & $\begin{array}{l}\text { Promedio de } \\
\text { gasto } \\
\text { acumulado } \\
\text { total/paciente }\end{array}$ & $\begin{array}{c}\text { Gasto } \\
\text { acumulado/mes }\end{array}$ & $\begin{array}{c}\text { Gasto } \\
\text { extradiálisis/mes }\end{array}$ & $\begin{array}{l}\text { Extradiálisis } \\
\% \text { del gasto } \\
\text { total }\end{array}$ & $\begin{array}{l}\text { Variación del } \\
\text { gasto extradiálisis } \\
\text { entre periodos }\end{array}$ \\
\hline \multirow[t]{4}{*}{ PAMI } & 1. ${ }^{\text {er }}$ trimes & 212 & $\$ 99,803.30$ & $\$ 33,267.77$ & $\$ 16,563.30$ & $50 \%$ & +3.4 veces \\
\hline & $4 .^{\circ}$ trimes & 2,152 & $\$ 375,595.35$ & $\$ 31,299.61$ & $\$ 4,891.15$ & $16 \%$ & \\
\hline & 1. ${ }^{\text {er }}$ semes & 358 & $\$ 148,044.94$ & $\$ 29,608.99$ & $\$ 13,849.91$ & $47 \%$ & +2.7 veces \\
\hline & $2 .^{\circ}$ semes & 2,278 & $\$ 371,917.71$ & $\$ 30,993.14$ & $\$ 5,103.18$ & $16 \%$ & \\
\hline \multirow[t]{4}{*}{ Otras OS } & 1. ${ }^{\text {er }}$ trimes & 102 & $\$ 118,362.69$ & $\$ 39,454.23$ & $\$ 16,886.70$ & $43 \%$ & +3.9 veces \\
\hline & $4 .^{\circ}$ trimes & 860 & $\$ 462,696.61$ & $\$ 38,558.05$ & $\$ 4,221.81$ & $11 \%$ & \\
\hline & 1. ${ }^{\text {er }}$ semes & 168 & $\$ 165,000.22$ & $\$ 33,000.04$ & $\$ 11,643.37$ & $35 \%$ & +2.7 veces \\
\hline & $2 .{ }^{\circ}$ semes & 909 & $\$ 456,100.45$ & $\$ 38,008.37$ & $\$ 4,322.85$ & $11 \%$ & \\
\hline
\end{tabular}

OS: obras sociales; PAMI: Programa Asistencial Médico Integral.

vs. 5.9 meses en $2 .^{\circ}$ semestre para las otras OS. En cuanto al promedio de episodios y días de internación, los eventos de afiliados al PAMI tuvieron menor duración global que en otras OS (5.7 vs. 8.5 días, $+32 \%$ ). Sin embargo, al registrarse menor cantidad de eventos, la estancia media fue mayor en los afiliados al PAMI (5.1 vs. 3.4 días/cama en el $1 .^{\text {er }}$ semestre), particularmente en los periodos precoces de tratamiento. En el análisis hacia dentro de cada cobertura en relación al periodo de tiempo analizado, los días/cama duplican su valor entre instancias precoces y alejadas en pacientes del PAMI (5.1 vs. 2.3 días/evento en $1 .^{\text {er }}$ y $2 .^{\circ}$ semestre, $+107 \%$ ). Como se citó previamente, los episodios de internación en otras OS no muestran cambios tan extremos y presentan menor cantidad de días/ evento (3.4 vs. 2.9 en el $1 .^{\text {er }}$ y $2^{\circ}$ semestre, $+17 \%$ ), quizás evidenciando un efecto de modalidad de cobertura (el pago por prestación supondría un incentivo similar para generar episodios de internación, independientemente de la gravedad de la patología o el momento de ocurrencia).

Al integrarse estos consumos ambulatorios y de internación con sus valores unitarios actualizados, se arribó a un valor de gasto adicional al monto mensual del módulo de diálisis. Hasta la realización de este estudio, este parámetro no había sido evaluado sobre grandes poblaciones con consumos reales en Argentina. El gasto extradiálisis por paciente representa en promedio un 17\% adicional para el PAMI y un $13 \%$ para las otras OS. Sin embargo, en función temporal, ese gasto es significativamente superior en los periodos más precoces (1. ${ }^{\text {er }}$ vs. $4 .^{\circ}$ trimestre y $1 .^{\text {er }}$ vs. $2 .^{\circ}$ semestre) (Tabla 5).

Las diferencias de este gasto extradiálisis son más marcadas entre periodos para los pacientes del PAMI:

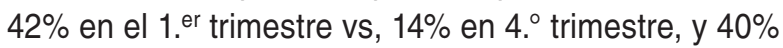
vs. $15 \%$ en el $1 .^{\text {er }}$ vs. $2 .^{\circ}$ semestre.

Al considerar la suma total de gasto incurrido por paciente para sostener su tratamiento dialítico y las demás prestaciones fuera de módulo necesarias para su atención clínica, puede arribarse a un valor medio mensual de gasto por afiliado, expresado en términos de gasto per cápita. Dado que los valores de módulo son fijos y varían en función del tiempo de permanencia en tratamiento, las diferencias en este valor per cápita estarán dadas por los gastos extramódulo por mes, los cuales dependen a su vez de la ocurrencia de episodios, el consumo de prácticas y su valorización. Siguiendo este análisis encontramos que el gasto promedio/paciente/mes extramódulo es entre 2 a 4 veces superior entre los periodos precoces (1. ${ }^{\text {er }}$ trimestre 0 semestre) que en los alejados $\left(4 .^{\circ}\right.$ trimestre $02 .^{\circ}$ semestre), evidenciando un mayor consumo de prestaciones y gastos cercanos al inicio del tratamiento dialítico en todas las coberturas analizadas.

\section{AnÁlisis multivariante}

A partir del mismo caso base seleccionado para el análisis de mortalidad, la regresión logística (Tabla 6) muestra una influencia significativa de la edad en la aparición de eventos de internación ( $25 \%$ de probabilidad 
Nefro Latinoam. 2019;16

Tabla 6. Regresión logística para odds ratios para frecuencia de eventos de internación

\begin{tabular}{|c|c|c|c|c|c|c|}
\hline CASO BASE (en negrita) & OR & SE & $\mathbf{z}$ & $p>z$ & \multicolumn{2}{|c|}{ IC 95\% } \\
\hline $\begin{array}{l}\text { Mujer } \\
\text { Hombre }\end{array}$ & 0.89 & 0.06 & -1.66 & 0.10 & 0.77 & 1.02 \\
\hline $\begin{array}{l}\text { Edad (menor } 50 \text { años) } \\
\text { Entre } 50 \text { y } 65 \text { años } \\
\text { Entre } 65 \text { y } 75 \text { años } \\
\text { Mayor de } 75 \text { años }\end{array}$ & $\begin{array}{l}1.13 \\
1.25 \\
1.44\end{array}$ & $\begin{array}{l}0.15 \\
0.15 \\
0.19\end{array}$ & $\begin{array}{l}0.96 \\
1.81 \\
2.68\end{array}$ & $\begin{array}{l}0.34 \\
0.05 \\
0.01\end{array}$ & $\begin{array}{l}0.88 \\
0.98 \\
1.10\end{array}$ & $\begin{array}{l}1.46 \\
1.59 \\
1.87\end{array}$ \\
\hline $\begin{array}{c}\text { No diabético } \\
\text { Diabético }\end{array}$ & 1.20 & 0.09 & 2.43 & 0.02 & 1.04 & 1.39 \\
\hline $\begin{array}{l}\text { Catéter transitorio } \\
\text { Fístula nativa } \\
\text { Fístula protésica } \\
\text { Catéter semipermanente }\end{array}$ & $\begin{array}{l}0.62 \\
0.61 \\
0.87\end{array}$ & $\begin{array}{l}0.05 \\
0.13 \\
0.23\end{array}$ & $\begin{array}{l}-5.61 \\
-2.34 \\
-0.51\end{array}$ & $\begin{array}{l}0.00 \\
0.02 \\
0.61\end{array}$ & $\begin{array}{l}0.52 \\
0.41 \\
0.52\end{array}$ & $\begin{array}{l}0.73 \\
0.92 \\
1.47\end{array}$ \\
\hline $\begin{array}{l}\text { Albuminemia menor de } 3 \mathrm{~g} / \mathrm{l} \\
\text { Entre } 3 \text { y } 4 \mathrm{~g} / \mathrm{l} \\
\text { Mayor a } 4 \mathrm{~g} / \mathrm{l}\end{array}$ & $\begin{array}{l}0.82 \\
0.77\end{array}$ & $\begin{array}{l}0.08 \\
0.08\end{array}$ & $\begin{array}{l}-2.02 \\
-2.50\end{array}$ & $\begin{array}{l}0.04 \\
0.01\end{array}$ & $\begin{array}{l}0.68 \\
0.63\end{array}$ & $\begin{array}{l}0.99 \\
0.95\end{array}$ \\
\hline $\begin{array}{l}\text { Hematocrito menor del } 22 \% \\
\text { Entre } 22 \text { y } 26 \\
\text { Entre } 26 \text { y } 30 \\
\text { Mayor a } 30\end{array}$ & $\begin{array}{l}0.95 \\
0.98 \\
1.00\end{array}$ & $\begin{array}{l}0.14 \\
0.13 \\
0.14\end{array}$ & $\begin{array}{l}-0.35 \\
-0.12 \\
0.02\end{array}$ & $\begin{array}{l}0.73 \\
0.91 \\
0.98\end{array}$ & $\begin{array}{l}0.72 \\
0.75 \\
0.77\end{array}$ & $\begin{array}{l}1.26 \\
1.28 \\
1.31\end{array}$ \\
\hline $\begin{array}{l}\text { Sin angor ni ICC } \\
\text { Angina persistente } \\
\text { Insuficiencia cardíaca }\end{array}$ & $\begin{array}{l}1.16 \\
1.06\end{array}$ & $\begin{array}{l}0.14 \\
0.10\end{array}$ & $\begin{array}{l}1.26 \\
0.63\end{array}$ & $\begin{array}{l}0.21 \\
0.53\end{array}$ & $\begin{array}{l}0.92 \\
0.88\end{array}$ & $\begin{array}{l}1.46 \\
1.27\end{array}$ \\
\hline
\end{tabular}

ICC: insuficiencia cardíaca congestiva; OD: odds ratio; SE: error estándar.

para pacientes entre 65-75 años y 44\% para mayores de 75 años); el diagnóstico de diabetes otorga un $20 \%$ de riesgo de internación en el primer año; el ingreso a diálisis con un catéter incrementa el riesgo de internación un $38 \%$ vs. la presencia de fístula arteriovenosa nativa o protésica; la albuminemia $<3 \mathrm{~g} / \mathrm{dl}$ incrementa un $23 \%$ el riesgo de internación; finalmente, las comorbilidades cardiovasculares se asociaron con un $6-16 \%$ de riesgo de internación, pero sin significación estadística.

\section{Discusión}

Las características de morbimortalidad elevada de los pacientes con ERCT incidentes en diálisis, con su consecutivo impacto en un incremento de eventos y costos asociados, representan una situación sanitaria ampliamente descripta a partir de estudios observacionales a gran escala en diversos países. El gasto creciente en tratamiento de reemplazo renal (TRR) ha llevado en la última década a revisar distintas consideraciones en torno a la real calidad de vida ofrecida a estos pacientes, así como los resultados en términos de ganancia de salud conseguida, medido por variables taxativas como la mortalidad o la aparición precoz de eventos (con su carga social, en la atención y en el financiamiento): ofrecer un beneficio supuesto por el reemplazo incompleto de una función orgánica como la renal mediante las TRR parece no ser la respuesta final a estos complejos dilemas éticos, sanitarios y de equilibrio económico.

El sistema de salud argentino presenta dificultades para establecer una comparación directa y basada en información explícita. Desde el financiamiento, las coberturas sanitarias tienen un conocimiento cabal del gasto incurrido en TRR (población de baja prevalencia relativa, con prestaciones moduladas de alto costo); sin embargo, las complicaciones derivadas de la alta morbilidad de estos pacientes y sus requerimientos de consumo de servicios y costos de atención no se encuentran disponibles de manera ordenada y disponible. El gasto real de un paciente en diálisis en Argentina (tratamiento dialítico y atención global de seguimiento y complicaciones) ha intentado ser estimado a través de distintas iniciativas de baja representatividad, utilizando bases de datos parciales. En ese sentido, este estudio constituye una propuesta inédita en el país, al intentar establecer la correlación entre la condición clínica de ingreso de los pacientes a diálisis, su cobertura de salud y la morbimortalidad asociada a eventos clínicos, consumo de prestaciones y gasto sanitario durante el periodo crítico del primer año posterior al ingreso. Se obtuvo una población de número significativo, con representación de distintas coberturas enrolada 
retrospectivamente a lo largo de 5 años. Las condiciones de ingreso (clínicas y de cobertura) se tomaron de variables seleccionadas del consolidado Registro SINTRA.SAN/INCUCAI, mientras que los consumos de prestaciones y el gasto reales se cogieron desde las bases transaccionales directas de los financiadores.

Mediante esta estrategia de recolección y ulterior análisis estadístico de la información, se buscó verificar (y en muchos casos comprobar) con base en datos reales lo siguiente:

- Los pacientes con ERCT incidentes tienen una alta mortalidad precoz, la cual tiene mayor impacto en aquellos con peores condiciones de ingreso (presencia de catéter al ingreso, comorbilidades, desnutrición).

- Los pacientes con peores condiciones clínicas presentan también mayores eventos precoces de internación y consumos de prestaciones médicas.

- El costo de atención es mayor en los primeros meses después del ingreso a diálisis.

- La mortalidad se asocia a características propias de distintas coberturas.

La muestra de población analizada refleja de manera significativa las características de los pacientes con ERCT incidentes en diálisis en el sistema de salud argentino: sobre esta base se buscó responder a interrogantes sobre el efecto de las condiciones clínicas y de cobertura al ingreso en la morbimortalidad, su consumo de servicios de salud y costos asociados en el primer año.

En línea con otros reportes de la literatura internacional ${ }^{13-16}$, los datos indican que la mortalidad es muy alta a los 90 y hasta 180 días posteriores al ingreso (2/3 de las muertes ocurren en el primer semestre) y luego disminuye en forma pronunciada trimestre a trimestre: en el caso del PAMI, la mortalidad del 1.er trimestre es del $42.3 \%$, y del $19.2 \%$ en promedio para los 3 trimestres siguientes, en las OSN es del $38.5 \%$ en el $1 .^{\text {er trimestre }}$ y el $20 \%$ el resto del periodo, y en las OSP, del $38.9 \%$ y $20.4 \%$, respectivamente. Contrastando, se observa que la mortalidad del 1. er trimestre en estos financiadores es incluso superior a lo hallado en estudios de referencia ${ }^{9}$. Estos resultados podrían implicar una selección de pacientes librada al método cuando sus condiciones clínicas no son debidamente mensuradas o corregidas, dejando sentado un precedente para poner en discusión el real beneficio que las TRR ofrecen por sí mismas en meros términos de «reemplazo funcional», sin considerar a los enfermos en la complejidad de sus comorbilidades clínicas y sociosanitarias. En cuanto a las características de los pacientes, los más añosos, con diagnóstico de diabetes o comorbilidad cardiovascular manifiesta (angina persistente o insuficiencia cardíaca) y menor nivel de albúmina $(<3 \mathrm{~g} / \mathrm{dl})$ tienen menor sobrevida que los pacientes sin estas características, y estas tasas se acentúan a lo largo del año de seguimiento. De igual forma, el catéter como acceso vascular (quizás el indicador más reconocible de llegada tardía al diagnóstico de la ERCT y la consulta nefrológica), influye en el riesgo de mortalidad de manera significativa.

En términos generales, tanto los consumos de prestaciones como los gastos relacionados a las mismas fueron mayores en los periodos más precoces después del ingreso a diálisis. Esto tuvo algunas diferencias en relación con los grupos de prácticas y eventos evaluados y al ser comparados entre financiadores por modalidad de cobertura. En cuanto al consumo de prácticas ambulatorias por paciente, se observó casi el doble de consultas y prácticas bioquímicas en el 1 er $^{\text {er }}$ semestre con respecto al $2 .{ }^{\circ}$ semestre. Analizando por

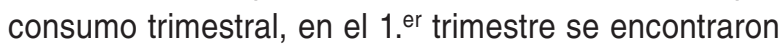
consumos mensuales 3 veces superiores en prácticas diagnósticas, y más de 7 veces en prácticas terapéuticas nomencladas respecto del $4 .^{\circ}$ trimestre.

La aparición de episodios de internación y su duración fueron los parámetros disponibles más ajustados para valorar el efecto de las condiciones al ingreso sobre la morbilidad. La cantidad de internaciones mostró una clara diferencia entre periodos: en el PAMI se observó una internación cada 1.5 meses en el 1.er trimestre vs. una internación cada 9.7 meses en el $4 .^{\circ}$ trimestre, una diferencia casi 7 veces superior en frecuencia de eventos; en las otras OS, la diferencia también es alta entre periodo, aunque no tan marcada. En el promedio de episodios y días de internación, los eventos de afiliados del PAMI tuvieron menor duración en días que en otras OS, pero al registrarse menor cantidad de eventos, la estancia media fue mayor, en particular en los periodos precoces. En este contexto, las diferencias observadas entre modalidad de pago de prestaciones permite suponer una mayor frecuencia de internaciones, pero de causas poco complejas en los afiliados de otras OS (pago por acto), las cuales mantienen una tendencia sin cambios en los periodos más alejados del momento de ingreso a diálisis. Por otra parte, en los afiliados PAMI (pago per cápita), las internaciones tienen menos frecuencia pero duración superior.

El gasto extradiálisis por paciente representa, en promedio, un $17 \%$ adicional para el PAMI y un $13 \%$ para las otras OS. Sin embargo, en función temporal, ese gasto es significativamente superior en los periodos más precoces y se hace notorio hacia el interior de cada cobertura: en términos generales el gasto promedio/ paciente/mes extramódulo es entre 3 a 4 veces superior 
entre los periodos precoces ( $1 .^{\mathrm{er}}$ trimestre 0 semestre) que en los alejados (4..$^{\circ}$ trimestre o $2 .^{\circ}$ semestre), lo que evidencia un mayor consumo de prestaciones y gastos cercanos al inicio del tratamiento dialítico. Finalmente, los factores asociados a la aparición de eventos de internación en el análisis multivariante fueron similares a aquellos que condicionaron la mortalidad: los pacientes $>65$ años, diabéticos, con catéter transitorio al ingreso y desnutrición grave tienen más riesgo de presentar internaciones en el primer año en diálisis.

\section{Conclusiones}

En la población de pacientes estudiada, hay altas tasas de mortalidad al año, con impacto en los periodos más precoces, con alto consumo de prestaciones y gasto financiero asociado, aunque con diferencias entre las frecuencias y montos al comparar entre financiadores de acuerdo a sus mecanismos de pago prestacional. Los pacientes que ingresan en peores condiciones al tratamiento (por probable llegada tardía a la consulta nefrológica, con acceso vascular transitorio y desnutrición grave), sumado a otras condiciones de riesgo (edad avanzada, comorbilidad cardiovascular o diabetes), presentan, además, mayores tasas de consumos de manera precoz. Quizás los sistemas de atención estén llegando tardíamente a dar la atención correcta a los pacientes, y poner a disposición altas cantidades de recursos (tanto para la TRR como extradiálisis) en este punto no logra mejorar los resultados sanitarios en términos de eficacia ni eficiencia.

\section{Conflicto de intereses}

Los autores declaran no presentar ningún tipo de conflicto de intereses que pueda afectar los resultados y los contenidos en relación con la presente publicación.

\section{Financiamiento}

Este artículo fue financiado por becas Salud Investiga «Dr. Abraam Sonis», categoría Estudio Multicéntrico, Dirección de Investigación para la Salud, Ministerio de Salud de la Nación (Argentina).

\section{Agradecimientos}

Los autores agradecen a Luciano del Blanco, Dora Amado, Natalia Grinblat, Guadalupe Montero, Federico
Lombardo, Karina Schiavino, Antonio Villamarín, Carolina Valenciaga, Ricardo Mastai, Augusto Vallejos y David Aruachan para la realización de este proyecto.

\section{Responsabilidades éticas}

Protección de personas y animales. Los autores declaran que para esta investigación no se han realizado experimentos en seres humanos ni en animales.

Confidencialidad de los datos. Los autores declaran que han seguido los protocolos de su centro de trabajo sobre la publicación de datos de pacientes.

Derecho a la privacidad y consentimiento informado. Los autores declaran que en este artículo no aparecen datos de pacientes.

\section{Bibliografía}

1. Brück K, Stel V, Fraser S, De Goeij M, Caskey F, Abu-Hanna A, Jager K. Translational research in nephrology: chronic kidney disease prevention and public health. Clin Kidney J. 2015;8(6)647-55.

2. Akbari A, Clase CM, Acott $P$, Battistella M, Bello A, Feltmate $P$, et al. Canadian Society of Nephrology Commentary on the KDIGO Clinical Practice Guideline for CKD Evaluation and Management. Am J Kidney Dis. 2015;65(2):177-205

3. Wingard R, Pupim L, Krishnan M, Shintani A, Ikizler T, Hakim R. Early Intervention Improves Mortality and Hospitalization Rates in Incident Hemodialysis Patients: RightStart Program. Clin J Am Soc Nephrol. 2007;2:1170-5.

4. Kent S, Schlackow I, Lozano-Kühne J, Reith C, Emberson J, Haynes R, et al. What is the impact of chronic kidney disease stage and cardiovascular disease on the anual cost of hospital care in moderate-to-severe kidney disease? BMC Nephrol. 2015;16:65.

5. Levin A. Consequences of late referral on patient outcomes. Nephrol Dial Transplant. 2000;15 Suppl 3:8-13.

6. Arora, P, Kausz A, Obrador G, Ruthazer R, Khan S, Jenuleson C, et al. Hospital utilization among chronic dialysis patients. J Am Soc Nephrol. 2000;11:740-6.

7. Sesso R, da Silva CB, Kowalski SC, Manfredi SR, Canziani ME, Draibe SA, et al. Dialysis care, cardiovascular disease, and costs in end-stage renal disease in Brazil. Int J Technol Assess Health Care. 2007 Winter;23(1):126-30.

8. Smart NA, Titus TT. Outcomes of early versus late nephrology referral in chronic kidney disease: a systematic review. Am J Med. 2011;124(11):1073-80.

9. Bradbury B, Fissell R, Albert J, Anthony M, Critchlow C, Pisoni R, et al. Predictors of Early Mortality among Incident US Hemodialysis Patients in the Dialysis Outcomes and Practice Patterns Study (DOPPS). Clin J Am Soc Nephrol. 2007;2:89-99.

10. Gillespie B, Morgenstern H, Hedgeman E, Tilea A, Scholz N, Shearon T, et al. Nephrology care prior to end-stage renal disease and outcomes among new ESRD patients in the USA. Clin Kidney J. 2015;8(6):772-80.

11. Marinovich S, Lavorato C, Bisigniano L, Hansen Krogh D, Celia E, Tagliafichi V, et al. Registro Argentino de Diálisis Crónica SAN-INCUCAI 2016. Sociedad Argentina de Nefrología e Instituto Nacional Central Único Coordinador de Ablación e Implante. Buenos Aires, Argentina; 2017.

12. Arce H. Organización y Financiamiento del Sistema de Salud en La Argentina. Medicina (Buenos Aires). 2012;72:414-8.

13. Kessler M, Frimat L, Panescu V, Briancon S. Impact of nephrology referral on early and midterm outcomes in ESRD: EPidemiologie de l'Insuffisance REnale chronique terminale en Lorraine (EPIREL): results of a 2-year, prospective, community-based study. Am J Kidney Dis. 2003;42:474-85.

14. Mau LW, Liu J, Qiu Y, Guo H, Ishani A, Arneson TJ, et al. Trends in patient characteristics and first-year medical costs of older incident hemodialysis patients, 1995-2005. Am J Kidney Dis. 2010;55(3):549-57.

15. Nig L, Chen F, Pisoni R, Krishnan M, Mapes D, Keen M, et al. Hospitalization risks related to vascular access type among incident US hemodialysis patients. Nephrol Dial Transplant. 2011;26:3659-66.

16. Kaysen G, Johansen K, Cheng S, Jin Ch, Chertow G. Trends and Outcomes Associated With Serum Albumin Concentration Among Incident. Dialysis Patients in the United States. J Ren Nutr. 2018;18(4):323-31. 